\title{
Ratio of preoperative atrial natriuretic peptide to brain natriuretic peptide predicts the outcome of the maze procedure in mitral valve disease
}

Masafumi Sato ${ }^{1}$, Akihito Mikamo ${ }^{1 *}$, Hiroshi Kurazumi ${ }^{1}$, Ryo Suzuki ${ }^{1}$, Masanori Murakami ${ }^{1}$, Toshiro Kobayashi ${ }^{1}$, Koich Yoshimura ${ }^{2}$ and Kimikazu Hamano'

\begin{abstract}
Background: Although the maze procedure is an established surgical treatment for eliminating atrial fibrillation $(A F)$, its efficacy in patients with mitral valve disease has remained unsatisfactory. A useful predictive marker for the outcome of the maze procedure is needed. The aim of this study was to investigate whether the preoperative ratio of atrial natriuretic peptide (ANP) to brain natriuretic peptide (BNP) reflects atrial fibrosis and can be used to predict the maze procedure outcome in patients with mitral valve disease.

Methods: A total of 23 consecutive patients who underwent the radial approach to the maze procedure combined with mitral valve surgery were included in this study and were divided into a sinus rhythm (SR) group $(n=16)$ and an AF group ( $n=7$ ) based on postoperative cardiac rhythm. Plasma samples were obtained at rest before the operation and were analysed for ANP and BNP levels. Atrial tissue samples taken during surgery were used to quantify interstitial fibrosis.

Results: The preoperative ANP-to-BNP ratio in the SR group was significantly higher than that in the AF group $(0.74+/-0.29$ vs. $0.42+/-0.28$, respectively; $p=0.025)$. Receiver operating characteristic $(R O C)$ curve analysis was used to identify factors that predict outcomes after the maze procedure. The area under the ROC curve for the ANP-to-BNP ratio (0.81) was greater than for any other preoperative factors. Moreover, the preoperative ANP-to-BNP ratio demonstrated a negative correlation with left atrial fibrosis $(r=-0.69 ; p=0.003)$.
\end{abstract}

Conclusions: The preoperative ANP-to-BNP ratio can predict maze procedure outcome in patients with mitral valve disease, and it represents a potential biomarker for left atrial fibrosis.

Keywords: Atrial fibrillation, Cardiac surgery, Fibrosis, Natriuretic peptides

\section{Background}

Atrial fibrillation (AF) is the most common cardiac arrhythmia, especially for patients with mitral valve disease. The presence of postoperative AF is a significant risk factor for postoperative mortality, stroke, and other thromboembolism and anticoagulant-related haemorrhage [1]. Therefore, the restoration of sinus rhythm (SR) is critical for patients with AF and mitral valve disease who underwent mitral valve surgery. The maze

\footnotetext{
* Correspondence: mikamo@yamaguchi-u.ac.jp

'Department of Surgery and Clinical Science, Division of Cardiac Surgery, Yamaguchi University Graduate School of Medicine, 1-1-1 Minami-Kogushi, Ube, Yamaguchi 755-8505, Japan

Full list of author information is available at the end of the article
}

procedure is an established surgical treatment for eliminating AF. The procedure reduces cardiovascular mortality and stroke and improves cardiac function [1,2]. Furthermore, its success rate for treating lone AF is higher than $90 \%$ [3]. However, only success rates between $60 \%$ and $90 \%$ have been reported with the maze procedure in patients with mitral valve disease $[4,5]$. Because the maze procedure has potential disadvantages, including prolonged operation and crossclamp times and the occasional need for implantation of a permanent pacemaker, performing this invasive procedure in patients who are less likely to receive a benefit should be avoided.

Atrial fibrosis plays an important role in developing and maintaining AF. Yoshihara et al. reported that the collagen

\section{Biomed Central}


volume in left atrial tissue was higher in AF than in SR [6]. However, previous risk factors for failure of the maze procedure, such as left atrial diameter (LAD), duration of $\mathrm{AF}$ and cardiothoracic ratio (CTR) [7,8], do not correlate well with atrial fibrosis [6,9]. Moreover, these risk factors do not fully predict the outcomes of the maze procedure. It has been reported that atrial natriuretic peptide (ANP) and brain natriuretic peptide (BNP) are related to atrial remodelling $[6,10]$. Furthermore, Mabuchi et al. reported that the ANP-to-BNP ratio was useful for predicting the recurrence of $\mathrm{AF}$ after direct current cardioversion in patients with mild chronic heart failure [11], which suggests that the ANP-to-BNP ratio may be useful for predicting the outcome of a maze procedure.

In the present study, we hypothesized that the preoperative ANP-to-BNP ratio more accurately predicts the outcome of a maze procedure in patients with mitral valve disease than the known risk predictors. Furthermore, we evaluated the ANP-to-BNP ratio as a biomarker for atrial interstitial fibrosis, which is a pathological process that has been associated with AF.

\section{Methods \\ Patients}

A total of 23 consecutive patients who had mitral valve disease (13 mitral regurgitation, 6 mitral stenosis and regurgitation, 4 mitral stenosis) and $\mathrm{AF}$ and underwent the radial approach to the maze procedure [12] at Yamaguchi University Hospital between April 2008 and December 2011 were examined. On the preoperative cardiac electrocardiogram, paroxysmal and chronic AF were documented in 1 and 22 patients, respectively. Left atrial enlargement was not used as an exclusion criterion. In addition, any patients with ischemic heart disease or chronic kidney disease were not included in this study. The cardiac rhythm after the maze procedure was assessed by electrocardiography $(n=21)$ or 24 hour electrocardiographic monitoring (Holter) $(n=2) 6$ months after surgery and represented the maze procedure outcome. Actually, SR was restored in 16 of 23 patients (69.6\%). To elucidate a predictive factor for maze procedure outcome, we divided the patients into SR $(n=16)$ and AF groups $(n=7)$ based on postoperative cardiac rhythm. Postoperatively, 2 patients with AF with slow ventricular response in the $\mathrm{AF}$ group and 1 patient with sick sinus symdrome in the SR group received permanent pacemakers. All protocols in the present study were approved by the Institutional Review Board of Yamaguchi University Hospital, and all patients gave written informed consent.

\section{Measurement of plasma ANP and BNP levels}

Blood samples were taken at rest before the operation. Plasma ANP concentration was measured with a chemiluminescent enzyme immunoassay for $\alpha$-human ANP (MI02
Shionogi ANP; Shionogi Co., Lts., Osaka, Japan) as previously described [13]. Plasma BNP concentration was measured with a chemiluminescent enzyme immunoassay for BNP (E Test TOSOH II (BNP); TOSOH Corporation, Tokyo, Japan) as previously described [14].

\section{Quantitative assessment of atrial fibrosis}

We examined interstitial fibrosis samples from the left atrium, left atrial appendage, right atrium and right atrial appendage of 16 patients in this study. The quantitative collagen assay (No. 9046, Chondrex, Inc., Redmond, WA, USA) was performed as previously described [15]. In brief, atrial tissue samples were fixed in formalin and embedded in paraffin. Ten-micrometer tissue sections were stained with $0.1 \%$ Sirius red and $0.1 \%$ Fast green dissolved in water saturated with picric acid for 30 minutes. The epicardium, endocardium and microvessels in the sections were then carefully removed under a microscope. The colour eluted from the sections with a mixture of $0.1 \mathrm{~N} \mathrm{NaOH}$ and methanol (1:1) was read in a spectrophotometer at $540 \mathrm{~nm}$ and $605 \mathrm{~nm}$. The net amounts of total collagen and non-collagenous proteins were calculated using absorbances at $540 \mathrm{~nm}$ and $605 \mathrm{~nm}$, respectively. The amount of total proteins was the sum of both absorbance values. Fibrosis was defined as follows: fibrosis $(\%)=$ (amount of collagen protein / amount of total proteins) $\times 100$.

\section{Statistical analysis}

Continuous data with was expressed as mean \pm standard deviation. Categorical variables were reported as frequencies. Pre- and postoperative data were analysed using the unpaired $t$-test and Fisher's exact test. The unpaired $t$-test was used for continuous variables with normal distribution. Fisher's exact test was used for categorical variables. Receiver operating characteristic (ROC) curve analysis was used to identify factors that predict the outcomes of a maze procedure. Associations between continuous variables were assessed using correlation and linear regression techniques. $\mathrm{P}<0.05$ was considered significant.

\section{Results}

\section{Preoperative ANP-to-BNP ratio as a predictive factor for maze procedure outcome}

To elucidate a predictive factor for maze procedure outcome, we compared preoperative variables between the SR $(n=16)$ and AF groups $(n=7)$. There were no significant differences in age, gender, CTR, left ventricular enddiastolic dimension (LVDd), ejection fraction (EF) and the preoperative ANP and BNP levels between two groups. In contrast, there was significant difference in LAD and the preoperative ANP-to-BNP ratio between the two groups $(\mathrm{p}=0.031, \mathrm{p}=0.025$, respectively). The preoperative ANPto-BNP ratio was not identified as an independent factor by the multivariate analysis $(\mathrm{p}=0.059)$, but compared to 
Table 1 Preoperative clinical characteristics of patients

\begin{tabular}{lccc}
\hline & $\begin{array}{c}\text { SR group } \\
(\mathbf{n = 1 6})\end{array}$ & $\begin{array}{c}\text { AF group } \\
(\mathbf{n = 7})\end{array}$ & p value \\
\hline Age (years) & $65.1+/-12.4$ & $70.4+/-5.6$ & $0.290^{\mathrm{a}}$ \\
\hline Gender (male/female) & $8 / 8$ & $2 / 5$ & $0.405^{\mathrm{b}}$ \\
\hline CTR $(\%)$ & $55.2+/-7.5$ & $61.9+/-5.9$ & $0.051^{\mathrm{a}}$ \\
\hline LVDd $(\mathrm{mm})$ & $53.8+/-8.9$ & $51.0+/-7.0$ & $0.554^{\mathrm{a}}$ \\
\hline LAD $(\mathrm{mm})$ & $51.5+/-10.6$ & $63.5+/-13.5$ & $0.031^{\mathrm{a}}$ \\
\hline EF $(\%)$ & $64.7+/-7.0$ & $68.7+/-7.3$ & $0.234^{\mathrm{a}}$ \\
\hline Preoperative creatinine $(\mathrm{mg} / \mathrm{dl})$ & $0.97+/-0.02$ & $1.10+/-0.18$ & $0.384^{\mathrm{a}}$ \\
\hline Preoperative ANP $(\mathrm{pg} / \mathrm{ml})$ & $120.1+/-67.3$ & $74.8+/-45.4$ & $0.141^{\mathrm{a}}$ \\
\hline Preoperative BNP $(\mathrm{pg} / \mathrm{ml})$ & $186.4+/-113.9$ & $191.9+/-72.2$ & $0.909^{\mathrm{a}}$ \\
\hline Preoperative ANP/BNP ratio & $0.74+/-0.29$ & $0.42 /-0.28$ & $0.025^{\mathrm{a}}$
\end{tabular}

${ }^{a}$ Unpaired t test. ${ }^{\mathrm{b}}$ Fisher's exact test.

$A F$ : atrial fibrillation; $A N P$ : atrial natriuretic peptide; BNP: brain natriuretic peptide; CTR: cardiothoracic ratio; $E F$ : ejection fraction; $L A D$ : left atrial dimension;

LVDd: left ventricular end-diastolic dimension.

the other factors, the ANP-to-BNP ratio demonstrated the most significant difference (Table 1).

To demonstrate the usefulness of the preoperative ANP-to-BNP ratio as a predictive factor for maze procedure outcome, ROC analysis was performed on the preoperative ANP-to-BNP ratio as well as ANP level, BNP level, CTR and LAD. The area under the curve (AUC) for CTR and LAD was 0.79 and 0.75 , respectively, indicating that CTR and LAD are also useful predictors of the outcome of a maze procedure, which is consistent with previous reports. Here, we found that the AUC for the preoperative ANP-to-BNP ratio was 0.81 or higher than AUC for ANP or BNP levels (Figure 1 and Table 2). Furthermore, the cutoff value for the preoperative ANP-to-BNP ratio to predict the restoration of sinus rhythm after a maze procedure was 0.41 (sensitivity $87.5 \%$, specificity $71.4 \%$ ). These findings indicate that the preoperative ANP-to-BNP ratio is the most useful for predicting the outcome of a maze procedure in patients with mitral valve disease.

\section{Correlation between the preoperative ANP-to-BNP ratio and atrial fibrosis}

Next, we investigated the correlation between the preoperative ANP-to-BNP ratio and atrial fibrosis. Figure 2 shows representative sections stained with $0.1 \%$ Sirius red and $0.1 \%$ Fast green in the left atrium (A, E), left atrial appendage $(B, F)$, right atrium $(C, G)$ and right atrial appendage tissues $(D, H)$ from patients with mild left atrial fibrosis (upper panel) and severe left atrial fibrosis (lower panel). In patients with mild and severe atrial fibrosis in the atrium, the degree of fibrosis was similar in the left atrial appendage, right atrium and right atrial appendage, but fibrosis (\%) in the left atrium was $6.2 \%$ in one of SR and $11.3 \%$ in one of AF, respectively. Furthermore, in patients with mild and severe atrial fibrosis, the preoperative ANP-to-BNP ratios were 0.79 and 0.15 , respectively. Interestingly, we found a negative correlation between the preoperative ANP-to-BNP ratio and fibrosis (\%) in the left atrium $(n=16, r=-0.69$; $\mathrm{p}<0.003$ ) (Figure 3A). In contrast, there was no correlation between the preoperative ANP-to-BNP ratio and fibrosis (\%) in the left atrial appendage, right atrium or right atrial appendage (Figure 3B, C and D). In addition, neither ANP nor BNP levels showed a correlation with fibrosis (\%) in any atrial tissues (data not shown).

We also observed that left atrial fibrosis (\%) in the AF group was significantly higher compared to that in the SR group (AF group, $8.7 \pm 2.4 \%$; SR group, $6.2 \pm 1.2 \%$; $\mathrm{p}=0.014$, Figure 4). In contrast, mean fibrosis (\%) in the left atrial appendage, right atrium and right atrial appendage in the AF group was slightly higher compared to that in the SR group (data not shown).

\section{Discussion}

This is the first demonstration of a relationship between the preoperative ANP-to-BNP ratio and maze procedure outcome in patients with mitral valve disease. Atrial remodelling is important in maintaining AF. Intra-atrial conduction block, which leads to small and numerous reentrant circuits, is caused by increased atrial fibrosis [16,17]. The maze procedure was developed as a surgical treatment for AF. In the maze procedure, interruption lines are placed to block the reentrant circuits. Therefore, an unsuccessful maze procedure may be the result of smaller reentrant circuits. The maze procedure cures AF in most patients with lone AF [3]. In contrast, the success rates of the maze procedure for patients with AF and mitral valve disease, which induces the progression of left atrial remodelling, have been reported to be between $60 \%$ and $90 \%$ and have remained unsatisfactory $[4,5]$. Because the progression of mitral valve disease is associated with structural changes

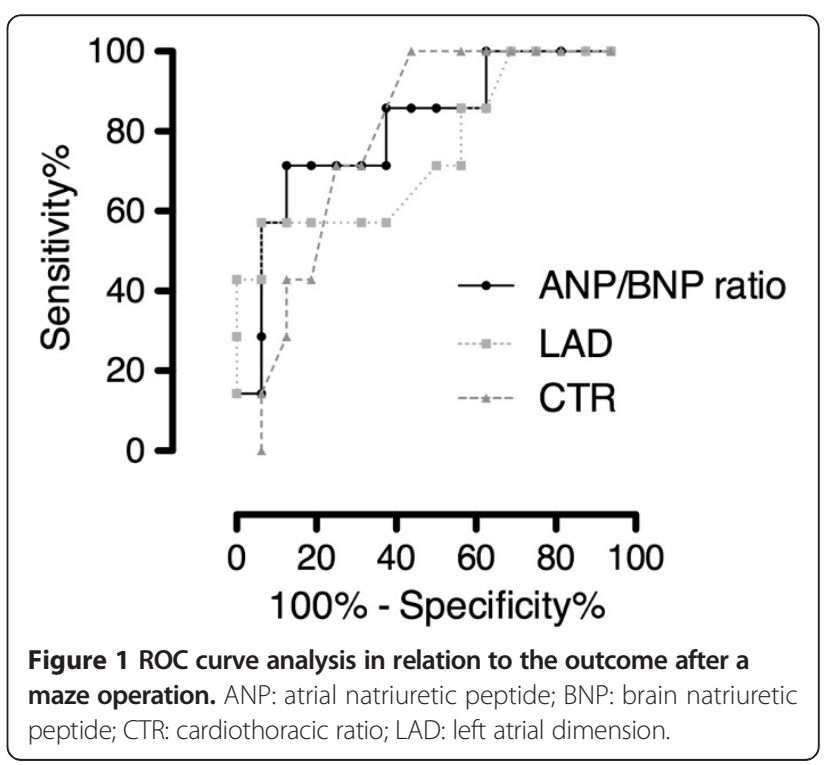


Table 2 Area under the curve for ANP-to-BNP ratio, ANP, BNP, CTR and LAD

\begin{tabular}{lccc}
\hline & AUC & p value & 95\% confidence interval \\
\hline Preoperative ANP/BNP ratio & 0.81 & 0.02 & $0.61-1.00$ \\
\hline Preoperative ANP & 0.73 & 0.08 & $0.50-0.96$ \\
\hline Preoperative BNP & 0.55 & 0.69 & $0.30-0.81$ \\
\hline CTR & 0.79 & 0.29 & $0.61-0.97$ \\
\hline LAD & 0.75 & 0.06 & $0.52-0.98$
\end{tabular}

ANP: atrial natriuretic peptide; AUC: area under the curve; BNP: brain natriuretic peptide; CTR: cardiothoracic ratio; $L A D$ : left atrial dimension.

of the atrial wall, such as fibrosis in atrial tissues, the efficacy of the maze procedure is theoretically limited in the patients with severe mitral valve disease. In addition, the maze procedure is an invasive treatment and has several disadvantages, including prolonged operation time. This has raised concerns that this procedure may result in increased mortality in patient subsets less likely to benefit from the procedure. To avoid an unnecessary or excessive procedure, a useful predictive marker for maze procedure outcome is necessary, especially for patients with mitral valve disease. Hence, we sought to identify a preoperative factor that predicts maze procedure outcome. Large LAD and increased CTR were previously reported as risk factors for recurring AF after a maze procedure $[7,8]$. Yoshihara et al. reported that plasma ANP level also predicts maze procedure outcome [6]. In this study, CTR and plasma ANP levels were not predictors of maze procedure outcome. This may be due to the small sample size in our current study. Despite the small sample size, we have succeeded in identifying the preoperative ANP-to-BNP ratio as the most powerful predictor of maze procedure outcome. In addition, we showed that the preoperative ANPto-BNP ratio was the best discriminator between patients with and without postoperative persistent AF (discriminator value $=0.41$ ). Using this cutoff value, the sensitivity and the specificity are $87.5 \%$ and $71.4 \%$ for predicting the restoration of sinus rhythm after a maze procedure. These results should lead to an increased success rate for the maze procedure. This strongly suggests that the preoperative ANP-to-BNP ratio is a promising tool for identifying the appropriate patients for the maze procedure.

We have also found that the preoperative ANP-to-BNP ratio is correlated with left atrial fibrosis. Although increased left atrial fibrosis is thought to be associated with the progression of mitral valve disease and failure of the maze procedure, the role of left atrial fibrosis in the development, recurrence and persistence of AF has not been fully elucidated. Saito and colleagues reported a correlation between the postoperative recurrence of $\mathrm{AF}$ and fibrosis in the left atrial appendage [18]. Kataoka et al. also reported that fibrosis in the left atrial appendage was significantly increased in patients in whom AF persisted after surgery [9]. Unfortunately, they did not assess left atrial interstitial fibrosis in their studies. In this study, we examined interstitial fibrosis in the left atrium, left atrial appendage, right atrium and right atrial appendage and found a considerable difference only in left atrial fibrosis between the SR and AF groups. Therefore, measuring left atrial fibrosis may be useful in determining the prognosis of patients with $\mathrm{AF}$ and mitral valve disease who underwent mitral valve surgery with a concomitant maze procedure. Moreover, the preoperative ANP-to-BNP ratio may also be a potential clinical biomarker for left atrial interstitial fibrosis.

Currently, the precise mechanism by which the preoperative ANP-to-BNP ratio is negatively correlated with left atrial fibrosis remains unknown. ANP is produced, stored and released by atrial cardiomyocytes in response to atrial stretch. It has been also reported that the decrease in plasma ANP is caused by the progression of atrial fibrosis [19]. This suggests that ANP by itself may be a potential biomarker for atrial fibrosis. Indeed, it has been reported that plasma ANP concentration inversely correlates with
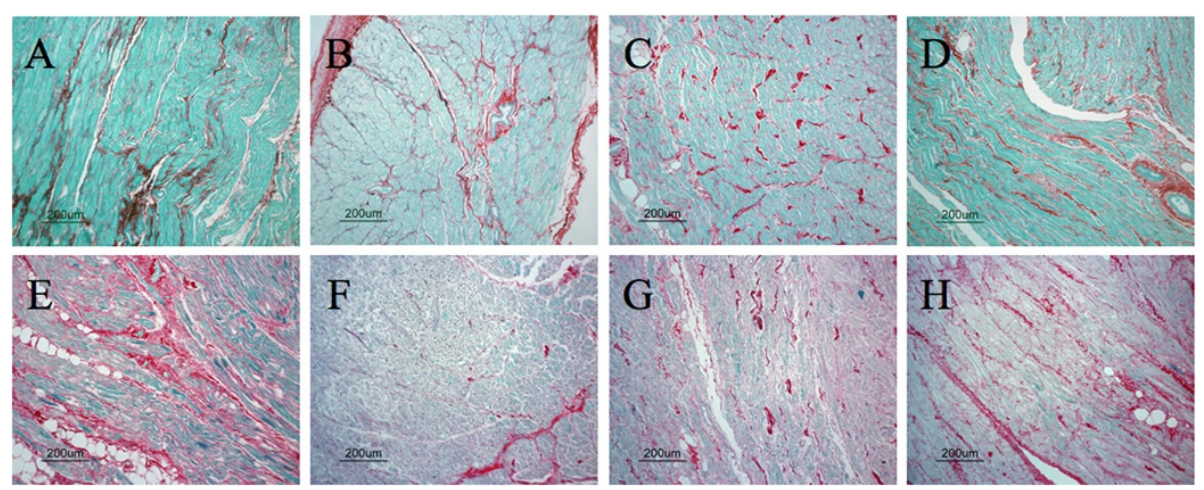

Figure 2 Representative sections stained with $0.1 \%$ Sirius red and $0.1 \%$ Fast green in the left atrium (A, E), left atrial appendage (B, F), right atrium $(C, G)$ and right atrial appendage $(D, H)$ of patients with mild left atrial fibrosis (upper panel) and severe left atrial fibrosis (lower panel). Scale bar=200 $\mu \mathrm{m}$. 

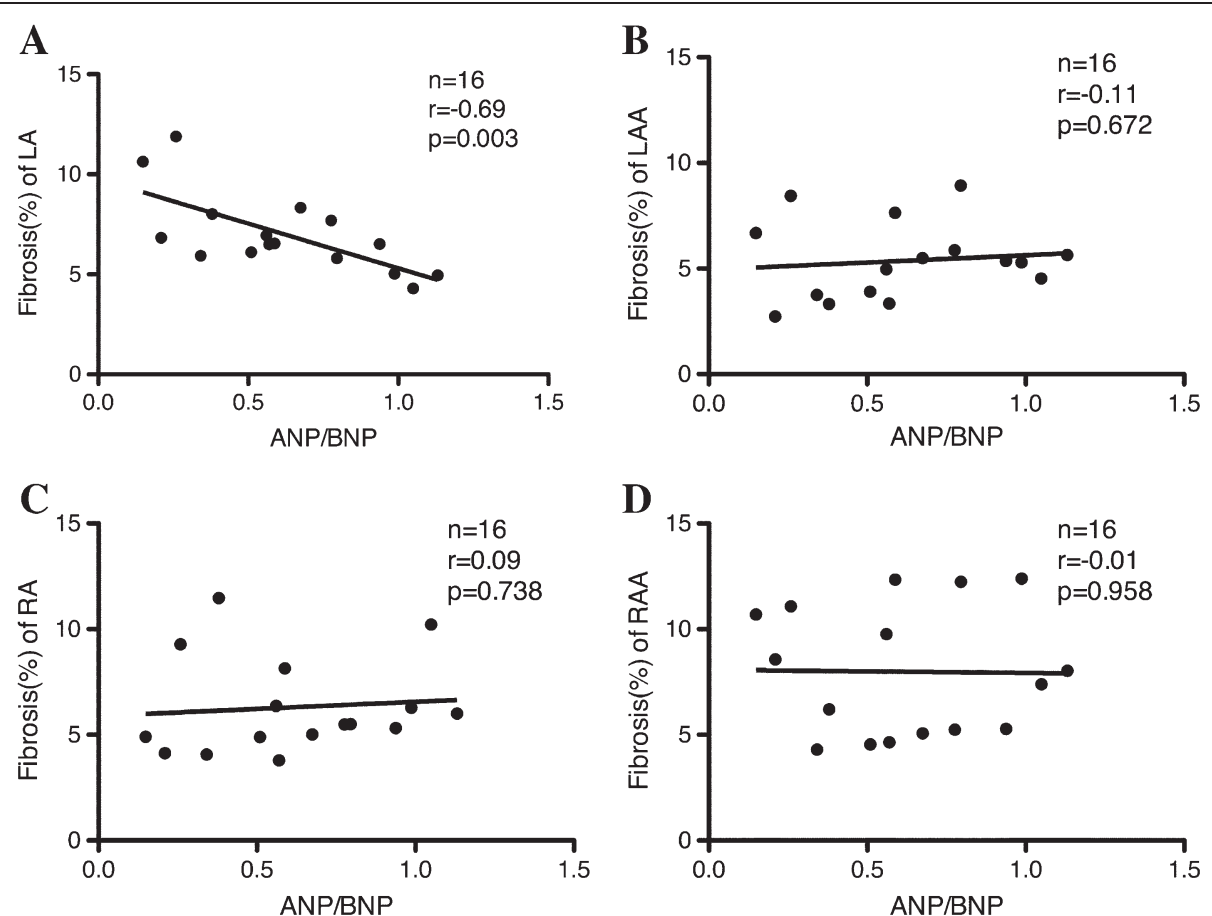

Figure 3 Correlations between plasma ANP-to-BNP ratio and fibrosis (\%) in the left atrium (A), left atrial appendage (B), right atrium (C) and right atrial appendage (D). ANP: atrial natriuretic peptide; BNP: brain natriuretic peptide.

left atrial collagen volume fraction and is related to the outcome of a maze procedure [6]. However, in patients with mitral regurgitation or stenosis, there is a concern that ANP may be influenced not only by atrial fibrosis but also by an increased pressure or volume load in the atria [20]. By contrast, BNP is mainly produced and secreted by the ventricles in response to ventricular overload [20]. In patients with mitral regurgitation, plasma BNP increases with the severity of mitral regurgitation [21]. Mitral regurgitation causes volume overload with left atrial enlargement and left ventricular remodelling and induces BNP activation [10]. In patients with mitral stenosis, the degree of mitral stenosis has been shown to correlate with plasma BNP level, which is mainly produced by right ventricular myocytes due to right ventricular overload [22]. There is also positive correlation between plasma BNP level and LAD in patients with mitral stenosis [23]. As a result, ANP secretion in a patient with mitral valve disease is reduced according to the progression of the LA remodelling and enhanced overload, and the secretion of BNP is increased due to LA remodelling in patients with mitral regurgitation and stenosis. Therefore, the ratio of ANP to BNP may represent the degree of atrial fibrosis, that is necessary to counter the effect of pressure and volume load on the secretion of ANP. The ratio of ANP to BNP may be a better biomarker for atrial fibrosis than ANP by itself. In this study, we clearly demonstrated that the preoperative ANP-to-BNP ratio is much more useful in predicting the outcome of a maze procedure and assessing left atrial fibrosis than either plasma ANP or BNP alone.

This study has some limitations. Although we observed a significant correlation between the preoperative ANP-to-BNP ratio and maze procedure outcome, the sample size was quite small. Further studies with larger sample sizes are needed to establish the usefulness of the preoperative ANP-to-BNP ratio. We assessed the

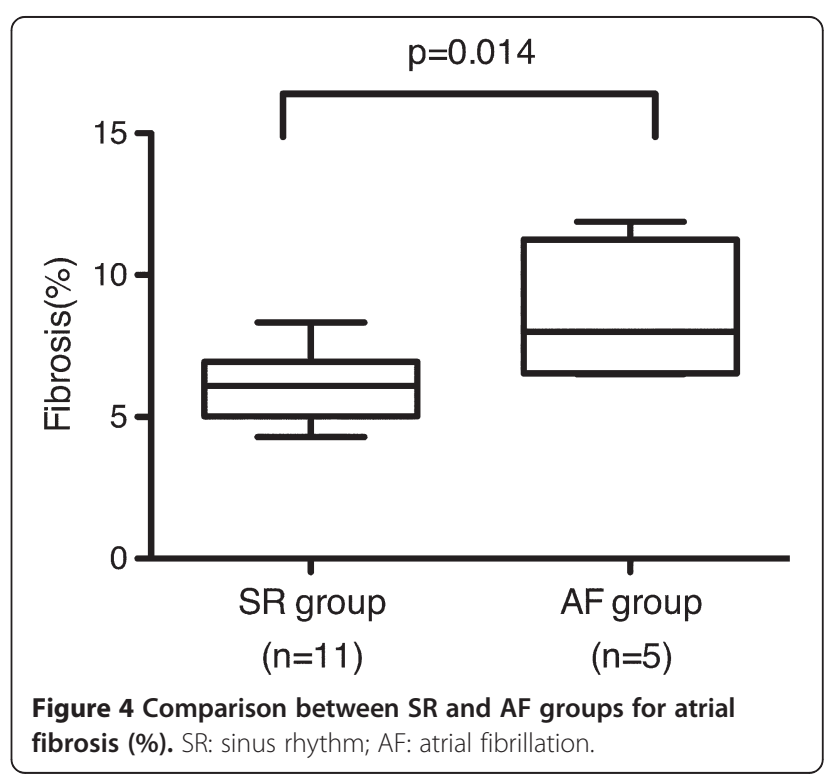


cardiac rhythm by electrocardiography in 21 patients and by 24 -hour electrocardio-monitoring in the remaining 2 patients. Therefore, the success rate after the maze procedure may be overestimated. In a previous study, when the use of electrocardiography was compared with 24-hour electrocardio-monitoring, the detection rate of AF recurrence differed by $5.5 \%$. However, the difference was not statistically significant [24]. In addition, even though we did not exclude such patients intentionally, no patients with ischemic heart disease or chronic kidney disease were enrolled in this study. BNP secretion was influenced not only by myocardial stretch but also by the ischemic insult [25]. It has also been reported that BNP is higher in patients with chronic kidney disease than in those without the disease [26]. These findings raise the possibility that the effectiveness of the preoperative ANP-to-BNP ratio may be decreased in patients with ischemic heart disease and/or chronic kidney disease. Therefore, the usefulness of preoperative ANP-to-BNP ratio in such patients should be examined in future studies.

\section{Conclusion}

In conclusion, our findings demonstrate that the preoperative ANP-to-BNP ratio correlates negatively with left atrial fibrosis, predicts the outcome of a maze procedure in patients with mitral valve disease and represents a new biomarker that helps to decide when to perform a maze procedure.

\section{Competing interests}

The authors declare that they have no competing interests.

\section{Authors' contributions}

MS developed study protocol, obtained data, analyzed data and wrote manuscript. AM developed the study protocol and provided critical revision of the manuscript. HK, RS, MM, TK, KY, and $\mathrm{KH}$ provided critical revision of the manuscript. All authors read and approved the final manuscript.

\section{Author details}

'Department of Surgery and Clinical Science, Division of Cardiac Surgery, Yamaguchi University Graduate School of Medicine, 1-1-1 Minami-Kogushi, Ube, Yamaguchi 755-8505, Japan. ${ }^{2}$ Faculty of Nursing and Nutrition, Yamaguchi Prefectural University, 3-2-1 Sakurabatake, Yamaguchi, Yamaguchi 753-0021, Japan

Received: 1 December 2012 Accepted: 27 February 2013

Published: 28 February 2013

\section{References}

1. Bando K, Kasegawa H, Okada Y, Kobayashi J, Kada A, Shimokawa T, Nasu M, Nakatani S, Niwaya K, Tagusari O, et al: Impact of preoperative and postoperative atrial fibrillation on outcome after mitral valvuloplasty for nonischemic mitral regurgitation. J Thorac Cardiovasc Surg 2005, 129(5):1032-1040.

2. Handa N, Schaff HV, Morris JJ, Anderson BJ, Kopecky SL, Enriquez-Sarano M: Outcome of valve repair and the Cox maze procedure for mitral regurgitation and associated atrial fibrillation. J Thorac Cardiovasc Surg 1999, 118(4):628-635.

3. Prasad SM, Maniar HS, Camillo CJ, Schuessler RB, Boineau JP, Sundt TM 3rd Cox JL, Damiano RJ Jr: The Cox maze III procedure for atrial fibrillation: long-term efficacy in patients undergoing lone versus concomitant procedures. J Thorac Cardiovasc Surg 2003, 126(6):1822-1828.

4. Wellens F, Casselman F, Geelen P, Brugada P, Van Praet F, De Geest R, Degrieck I, Vanermen H: Combined atrial fibrillation and mitral valve surgery using radiofrequency technology. Semin Thorac Cardiovasc Surg 2002, 14(3):219-225.

5. Gillinov AM, Saltman AE: Ablation of atrial fibrillation with concomitant cardiac surgery. Semin Thorac Cardiovasc Surg 2007, 19(1):25-32.

6. Yoshihara F, Nishikimi T, Sasako Y, Hino J, Kobayashi J, Minatoya K, Bando K, Kosakai Y, Horio T, Suga S, et al: Plasma atrial natriuretic peptide concentration inversely correlates with left atrial collagen volume fraction in patients with atrial fibrillation: plasma ANP as a possible biochemical marker to predict the outcome of the maze procedure. J Am Coll Cardiol 2002, 39(2):288-294.

7. Bando K, Kobayashi J, Kosakai Y, Hirata M, Sasako Y, Nakatani S, Yagihara T, Kitamura S: Impact of Cox maze procedure on outcome in patients with atrial fibrillation and mitral valve disease. J Thorac Cardiovasc Surg 2002, 124(3):575-583.

8. Isobe F, Kawashima Y: The outcome and indications of the Cox maze III procedure for chronic atrial fibrillation with mitral valve disease. J Thorac Cardiovasc Surg 1998, 116(2):220-227.

9. Kataoka T, Hamasaki S, Inoue K, Yuasa T, Tomita K, Ishida S, Ogawa M, Saihara K, Koriyama C, Nobuyoshi M, et al: Left atrium volume index and pathological features of left atrial appendage as a predictor of failure in postoperative sinus conversion. J Cardiol 2010, 55(2):274-282.

10. Detaint D, Messika-Zeitoun D, Avierinos JF, Scott C, Chen H, Burnett JC Jr, Enriquez-Sarano M: B-type natriuretic peptide in organic mitral regurgitation: determinants and impact on outcome. Circulation 2005, 111(18):2391-2397.

11. Mabuchi N, Tsutamoto T, Maeda K, Kinoshita M: Plasma cardiac natriuretic peptides as biochemical markers of recurrence of atrial fibrillation in patients with mild congestive heart failure. Jpn Circ J 2000, 64(10):765-771.

12. Nitta T, Lee R, Schuessler RB, Boineau JP, Cox JL: Radial approach: a new concept in surgical treatment for atrial fibrillation I. Concept, anatomic and physiologic bases and development of a procedure. Ann Thorac Surg 1999, 67(1):27-35.

13. Ken-ichi S, Tsutayo H, Akiko T, Toshio S, Hitoshi T, Toshihiko F: Evaluation of automated chemiluminescent enzyme imunoassay analyzer exclusive kit "MIO2 Shionogi ANP". Jpn J Med Pharm Sci 2007, 57(6):901-906.

14. Yoshitomo N, Emi F, Hiroshi S, Junichi M, Yutaka I: Evaluation of plasma brain natriuretic peptide concentration used by AIA-360. Jpn J Med Pharm Sci 2004, 52(3):397-402.

15. Lopez-De Leon A, Rojkind M: A simple micromethod for collagen and total protein determination in formalin-fixed paraffin-embedded sections. J Histochem Cytochem 1985, 33(8):737-743.

16. Li D, Fareh $S$, Leung TK, Nattel S: Promotion of atrial fibrillation by heart failure in dogs: atrial remodeling of a different sort. Circulation 1999, 100(1):87-95.

17. Allessie M, Ausma J, Schotten U: Electrical, contractile and structural remodeling during atrial fibrillation. Cardiovasc Res 2002, 54(2):230-246.

18. Saito T, Tamura K, Uchida D, Togashi M, Nitta T, Sugisaki Y: Histopathological features of the resected left atrial appendage as predictors of recurrence after surgery for atrial fibrillation in valvular heart disease. Circ J 2007, 71(1):70-78.

19. Maeda K, Tsutamoto T, Wada A, Hisanaga T, Nishimura T, Yamada H, Sasaki $Y$, Kinoshita M: Low dose synthetic human atrial natriuretic peptide infusion in a patient with mitral stenosis and severe pulmonary hypertension. Jpn Circ J 1999, 63(10):816-818.

20. Yoshimura M, Yasue H, Okumura K, Ogawa H, Jougasaki M, Mukoyama M, Nakao K, Imura H: Different secretion patterns of atrial natriuretic peptide and brain natriuretic peptide in patients with congestive heart failure. Circulation 1993, 87(2):464-469.

21. Sutton TM, Stewart RA, Gerber IL, West TM, Richards AM, Yandle TG, Kerr AJ: Plasma natriuretic peptide levels increase with symptoms and severity of mitral regurgitation. J Am Coll Cardiol 2003, 41(12):2280-2287.

22. Eryol NK, Dogan A, Ozdogru I, Inanc MT, Kaya MG, Kalay N: The relationship between the level of plasma B-type natriuretic peptide and mitral stenosis. Int J Cardiovasc Imaging 2007, 23(5):569-574.

23. Arat-Ozkan A, Kaya A, Yigit Z, Balci H, Okcun B, Yazicioglu N, Kucukoglu S: Serum N-terminal pro-BNP levels correlate with symptoms and 
echocardiographic findings in patients with mitral stenosis. Echocardiography 2005, 22(6):473-478.

24. Ad N, Henry L, Hunt S, Barnett S, Stone L: The Cox-Maze III procedure success rate: comparison by electrocardiogram, 24-hour holter monitoring and long-term monitoring. Ann Thorac Surg 2009, 88(1):101-105.

25. Morrow DA, Braunwald E: Future of biomarkers in acute coronary syndromes: moving toward a multimarker strategy. Circulation 2003, 108(3):250-252.

26. Marumoto K, Hamada M, Hiwada K: Increased secretion of atrial and brain natriuretic peptides during acute myocardial ischaemia induced by dynamic exercise in patients with angina pectoris. Clin Sci (Lond) 1995 88(5):551-556.

\section{doi:10.1186/1749-8090-8-32}

Cite this article as: Sato et al:: Ratio of preoperative atrial natriuretic peptide to brain natriuretic peptide predicts the outcome of the maze procedure in mitral valve disease. Journal of Cardiothoracic Surgery 2013 8:32

\section{Submit your next manuscript to BioMed Central and take full advantage of:}

- Convenient online submission

- Thorough peer review

- No space constraints or color figure charges

- Immediate publication on acceptance

- Inclusion in PubMed, CAS, Scopus and Google Scholar

- Research which is freely available for redistribution 